\title{
Online Electronic Examination System
}

\author{
Ali. Abdallah \\ Computer \& Communication Department, Islamic University Of Lebanon \\ AutoRoute Khaldeh, Khaldeh Lebanon
}

\begin{abstract}
The Online Electronic Examination System is a new System that helps universities, colleges and schools to have secure and automated exams with perfect results. The system is computer based, an application based on client-server architecture. Teachers deliver an exam to the system, the system reconstruct the exam format and distribute it among the students registered for the exam, the results of the exam will be directly obtained and saved.
\end{abstract}

\section{Introduction.}

Recently, a great buzz has been surrounding $\quad$ E-Learning standards. But what are these ELearning "standards", and what do they mean to the people designing and implementing E-Learning initiatives? Today, it may translate into confusion and a daunting level of research and mental investment. But in the future, this work should give E-Learning the flexibility we take for granted in other applications. E-Learning standards are the vehicle that will bring flexibility to content and infrastructure solutions. They open the door for more sensible and coherent ways to package learning content and resources for both students and developers alike. In E-Learning, the Instructional Management System (IMS) Metadata committee is such a specification group. This group has been clarifying issues such as: How should ELearning content be tagged? What fields should be required? and How can this information be communicated?

As the specification and adoption process continues, designers and implementations of E-Learning programs can expect a number of specific and significant capabilities:

Content portability - When content has been separated from a proprietary delivery system, the organization can consolidate, organize and track their E-Learning initiatives in the LMS of their choice. Because this is true for both third-party custom content, corporations will have greater flexibility and lower switching costs. Granularity - The new specifications support the learning object methodology, allowing for smaller and more timely units of information. Learning objects adds "just enough" to "just-in-time" learning.

Interoperability - Application interoperability starts where different E-Learning applications can share content and tracking data. But even more exciting, these specifications open up the possibility for different types of applications to swap and access content. A CRM application, for example, should be able to access learning objects from an E-Learning application, supporting the users more seamlessly.

E-Learning and knowledge management - The first and most obvious use of interoperability would be ELearning and knowledge management applications.

On one hand, an instructor should be able to access an item stored in a knowledge management system, and include it in a learning track. On the other hand, what is content - especially custom content - but knowledge that has been captured and structured for transfer to others? However, without a common foundation, like XML, and a common framework, such as IMS metatags, the effort of mapping data through XML interpreters will prove to be daunting. Fortunately, all of the benefits of the specifications efforts - portability, granularity, and interoperability - should form the basis for this convergence.

\section{Objectives.}


The Online Electronic Examination System is based on a scientific research, and designed to make use of the distributed application in order to facilitate the examination process, and secure the results. The system is developed to offer the teachers efficient tools, to monitor the examination process, and control all the stages of the exam. The system also offers students great methods to react with their exam, and make use of the available technologies.

The system supports the educational institutes with such a great technology, and save time and efforts in delivering and correcting exams. It also offers a great speed and accurate results with acceptable security and privacy level.

The goal of the system is to help the educational institutes during their examination process and make use of the technology issued by the E-Learning techniques to maintain maximum efficiency and accuracy. The scope of the system is to deliver electronic exam sheets among the students, the students make their answers, the system then correct the electronic exam sheets submitted by the students and produces marks immediately, and save a copy of the marks with the date and time of the exam in its database for later processes.

\section{OEES General Design.}

The Online Electronic Examination System (OEES) is based on three essential parts witch interact with each others to perform the examination process and produce the results starting from loading the exam and ending with generating the final exam result.

The Administrator receives the exam document from the teacher in a doc format. Each exam has a title, refers to a course, has a type and got a maximum mark range. Example: Math test, partial exam, over 40.

Using the Admin Application (Java based application), the Administrator loads the exam into the system database.

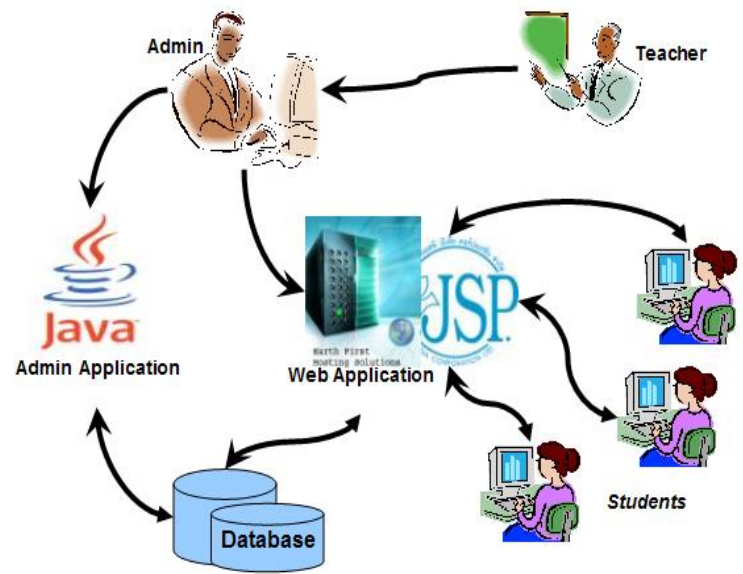

Figure 1. The General block Diagram for the Online Electronice Examination System (OEES)

students, using the Web Server (Examination Website), through the local area network (LAN), can log on the server, choose the specified exam, check whether it is available or not, and whether they are registered for this exam or not, then they can access the exam sheet, do their exam and receive their grades (results) immediately .

The Examination process starts from the teacher's document exam, the administrator using his special java application loads the exam to the database with all its required information, from the other side, students access the OEES web site, log into the system, load the exam sheet and do their exams. When finished, the system checks the answers and generates reports for the students concerning their grades. [Figure 1].

\section{System Analysis and Design:}

\subsection{Three Tier Application.}


Through the appearance of Local-Area-Networks, PCs came out of their isolation, and were soon not only being connected mutually but also to servers. Client/Server-computing was born.

Servers today are mainly file and database servers; application servers are the exception. However, databaseservers only offer data on the server; consequently the application intelligence must be implemented on the PC (client).

\subsubsection{Client-tier.}

Client-tier is responsible for the presentation of data, receiving user events and controlling the user interface. The actual business logic has been moved to an application-server. Today, Java-applets offer an alternative to traditionally written PC-applications [Figure 2].

\subsubsection{Application-server-tier.}

This tier is new, i.e. it isn't present in 2-tier architecture in this explicit form. Business-objects that implement the business rules "live" here, and are available to the client-tier. This tier protects the data from direct access by the clients [Figure 2].

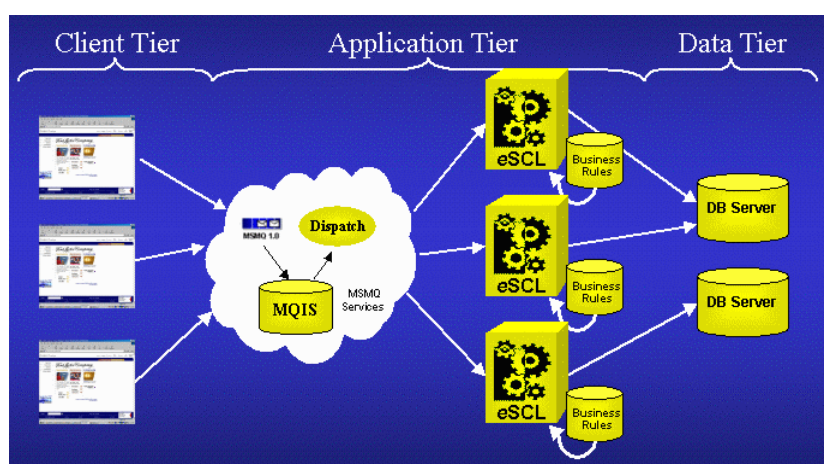

Figure 2. The three tier application representing its parts (client, application and data).

\subsubsection{Data-server-tier.}

This tier is responsible for data storage. Besides the widespread relational database systems, existing legacy systems databases are often reused here.

It is quite easily possible to run all three tiers on one and the same (physical) machine. The main importance is that the system is neatly structured, and that there is a well planned definition of the software boundaries between the different tiers [Figure 2].

\subsection{Exam sheet format.}

Exam sheets will be interpreted by the Admin Application (Java Application), this application is responsible for analyzing the exam file prepared by the teacher, in order to construct the exam file and records after checking all the required information, so that, the sheet must respect a specific designed format. Teachers must be careful while writing their exam document.

Each Information concerning a Unique exam must start with the '*' character.

All Fields are Mandatory (can't contain Null values).

Duration: Exam duration in minutes.

Exam sheet must be like following:

*CourseId: Net

*TeacherId: 1 
*Duration: 120

*Title: Network Exam

*Major: cce_f

*Type: Final

*AcYearId: 5

*Year: 3

*Semester: Fall

The admin application in fact generates a doc file containing information about the exam. Each teacher is obliged to follow up the format and the design of the exam sheet generated by the system, and fill up all the required information concerning his exam. This process facilitates the job of the teacher in preparing his exam, and minimizes the error rate in building up the exam file, and in processing the information to reconstruct the exam on the web server

Every Question must start with 'Q' followed by question number, then ':' then Question Type followed by ':' and finally Question Grade.

Question Title begins with a dash '-'.

Question Types are:

TF: True or False Question.

MC: Multiple Choices Question.

BF: Blank Fields Question.

\subsubsection{True or False Question (TF):}

Only one answer (right answer) is inserted after a point '.' like following (t: means True, f: means False):

Q1:TF:3

-Answer of $1+1$ is 2

.$t$

\subsubsection{Multiple Choices Question (MC):}

Q2:MC:3

-What expressions give 4 ?

. $3+1: \mathrm{t}$

$.2+3: \mathrm{f}$

$.2 * 2: \mathrm{t}$

$.4+0: \mathrm{t}$

\subsubsection{Blank Fields Question (BF):}

\section{Q3:BF:2}

-IUL Building in

.Khaldeh

After processing the exam file prepared by the teacher, the system generates the exam to the web server in the form of database records, where information about the exam and the questions with their types are stored.

Now the students are able to apply for the exam, referring to their identification numbers and some other information which is required to apply for the exam.

After finishing the exam, the answers will be collected for every student, saved, corrected, and then the system generates the final result with the grade.

\begin{tabular}{|c|c||c|c|c||c|}
\hline Value & Letter & Value & Letter & Value & Letter \\
\hline $100,99,98$ & A+ & $\mathbf{9 7 , 9 6 ,}$ & $\mathrm{A}$ & $\mathbf{9 3 , 9 2 ,}$ & $\mathrm{A}-$ \\
& & $\mathbf{9 5 , 9 4}$ & & $\mathbf{9 1 , 9 0}$ & \\
\hline
\end{tabular}




\begin{tabular}{|c|c||c|c|c||c|}
\hline $89,88,87$ & B+ & $\begin{array}{r}86,85, \\
84,83\end{array}$ & B & $\begin{array}{c}82,81, \\
80\end{array}$ & B- \\
\hline $79,78,77$ & C+ & $\begin{array}{r}76,75, \\
74,73\end{array}$ & C & $\begin{array}{c}72,71, \\
70\end{array}$ & C- \\
\hline $69,68,67$ & D+ & $\begin{array}{r}66,65, \\
64,63\end{array}$ & D & $\begin{array}{c}62,61, \\
60\end{array}$ & D- \\
\hline ELSE & F & \multicolumn{4}{|c|}{} \\
\hline
\end{tabular}

Table 1: This table shows the method of transforming Numerical values of grade into American System values (Alphabetic Letters).

The result is calculated and generated in both the American system grading and the numerical grade system [Table 1].

\section{Relational Database Design.}

\subsection{Relational Database.}

It's a complete database including many tables and relations to build the data part of the system, so this database contains all information concerning students, teachers, courses, exams, grades, questions, answers etc...

\subsection{Business rules:}

- $\quad$ One Major has 1 or many Students; Student can register in only 1 Major.

- Every CurrentCourse is defined by: 1 Course, 1 Year, 1 AcademicYear, 1 Semester, 1 Teacher for 1 Major.

- Every CurrentCourse may have 1 or many exams; Exam must be for a CurrentCourse.

- Every Student must take a Grade for an Exam.

- One Exam contains 1 or many Questions;

- Question may have 1 or many Answers.

The database contains 22 entities witch are listed below:

Admin: This table contains all information concerning Examination System Administrators, this table is responsible for administrators $\log$ in, and offers them different privileges referring to their positions and access types.

Student: This table contains information about students registered in the System, with a login passport to the web page to access the system and do exams.

Teacher table: This table contains information about teachers registered to the System with their degrees and professions.

Major: This table contains information about majors registered to the System.

Year: This table contains information about years registered to the System.

AcademicYear: This table contains information about academic years registered to the System.

Semester: This table contains information about semesters registered to the System.

Course: This table contains information about Courses registered to the System.

CurrentCourse: This table contains information about a course in a specific year, for a specific academic year and semester, given by a specific teacher. The current course table carries records about the active courses.

Exam: This table contains all exams loaded through the java application.

Grade: This table contains the grades of each student in each exam.

Question: This table contains all questions of all exams loaded from the java application.

QuestionFile: Some questions may contain one or many graphs, pictures or even sound clips, this entity allow system to store file paths to be loaded. TFAnswer: True Or False Answer (Indicator), this table contains information about questions, telling if the correct answer is true or false.

BFAnswer: Blank Field Correct Answers, it contains the correct answer(s) of a given "Fill in the Blank" question. 
MCAnswer: Multiple Choice Options (Choices), these choices can be true or false, every row in this table has a true/false indicator.

Login: Similar System needs for sure certain limits of security, this table contains information about every "Login" operation made (Login Logs).

Message: This table contains all messages posted by users/visitors through the Web Site.

Guest: This table contains only 1 field with 1 row; it indicates the total number of visitors of the web site (Guest Counter).

McCorrection: This table is considered temporary and hidden, it is helpful in the correction of Multiple Choice questions.

SecretQuestion: this table helps students who forgot their passwords, if they have a record in this table, they can get their passwords back again [Figure 3].

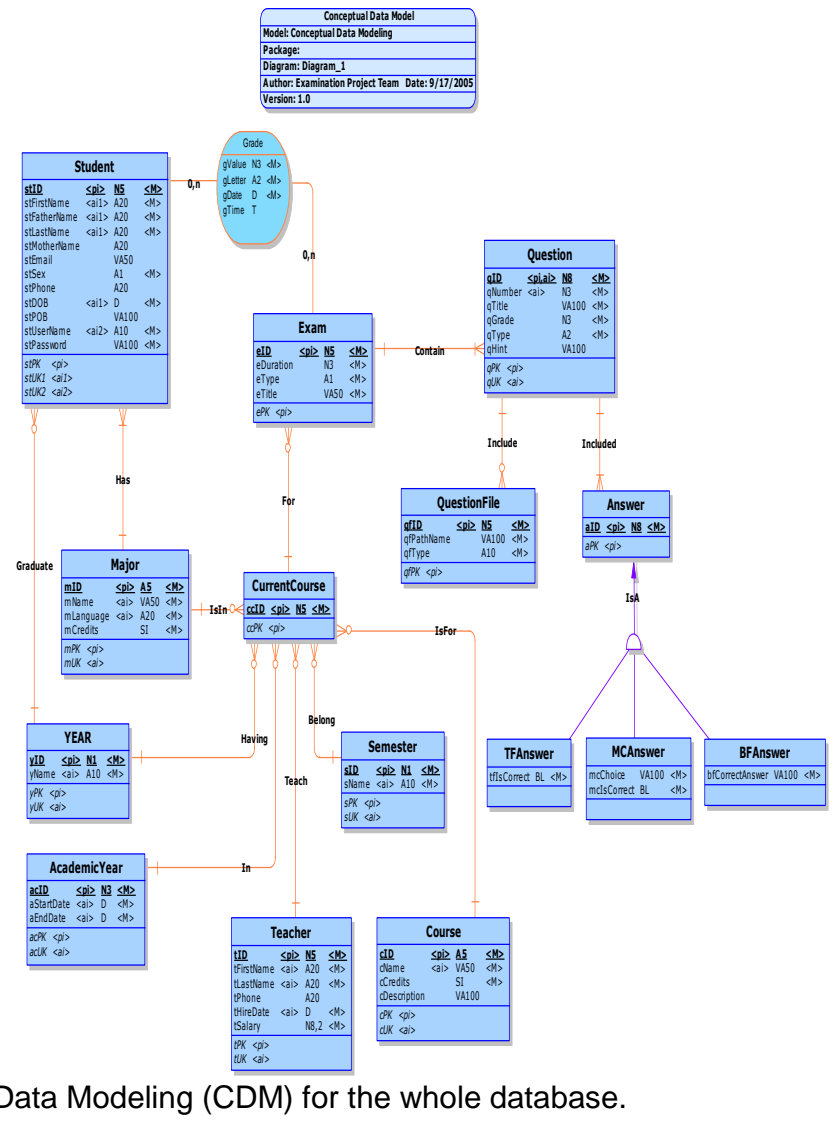

Figure 3. Conceptual Data Modeling (CDM) for the whole database.

\section{Admin application Design and function}

The Admin application can be used by Administrators only (People having a record in the table "Admin" of the Examination Database).

This application allow Admin to manage some database tables using a simple GUI (Graphical User Interface), like adding/deleting/updating students, teachers, courses, exams, questions, answers, messages and so on...

Class SqlAnalyser: A Java GUI Frame allowing Administrator to perform his own SQL SELECT statement and brownsing results on screen.

Class OdbcToSQL: A Java class including the connection to database through Odbc Drivers.

Class ESInterpreter: It's the most active class of the application, a GUI Frame allowing Administrator to give the file path of the exam sheet, and load exam questions and answers into database. This class contains kind of decoder for the Exam Sheet Keywords 


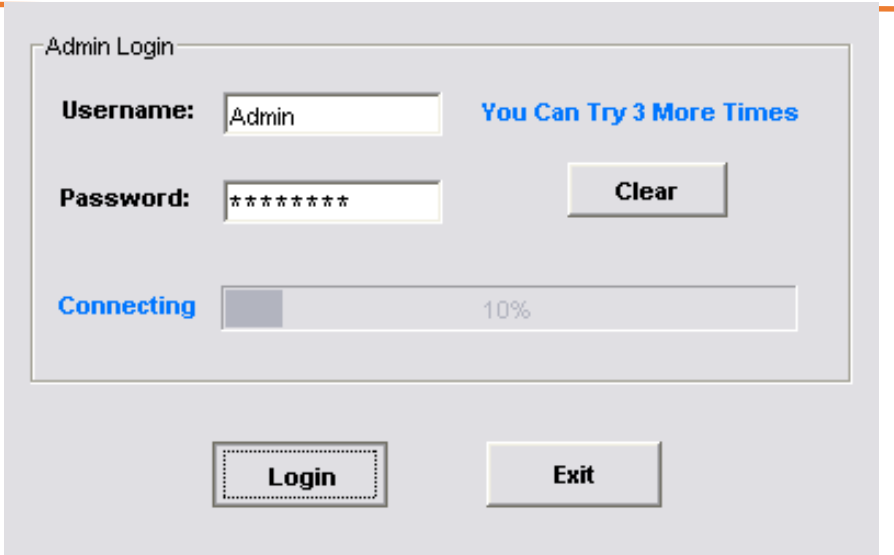

Figure 4. loging in form for the Admin application where user name and passwords are required.

The log in form allows the administrator to access the administration application where user names and passwords are required. The administrator then is able to brows any enformation and make any changes including creating new exam. The administrator can add new Exam, configure an existing one, update information to students, courses, marks etc.[Figure 4]

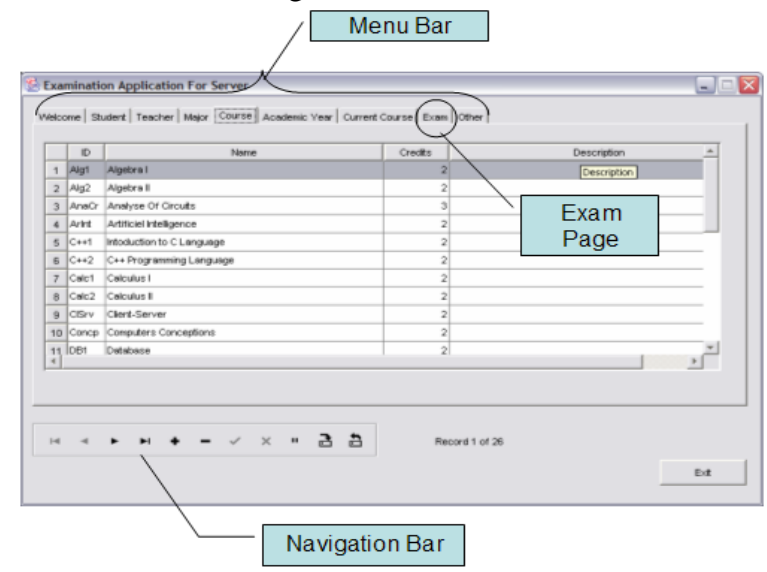

Figure 5. control form used by the administrator to control the database data.

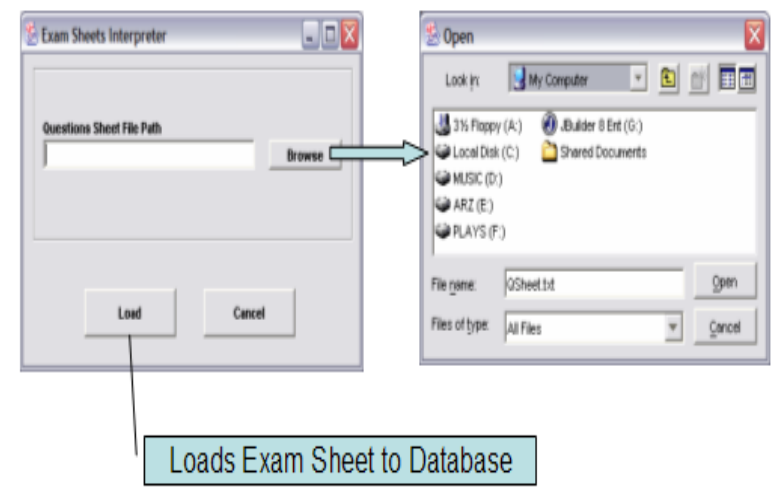

Figure 6. administrators can load the .doc file exam to be decoded and processed

In order to add a new exam, the administrator, using the exam form, can brows the exam file prepared by the teacher, then load the file to the application where decoding process starts. The application reads the 
.doc file, decode its content and insert to the desired tables all the information needed in order to create a new exam [Figure 6].

The administrator checks the file before loading it, the system interns, notify the administrator for any error and ask him to correct it. When the process is sucsseded, a report is generating to confirm all the information previously processed. The administrator confirms for the information about the exam after making necessary changes befor creating the record of the exam .

\section{Web Application.}

The most important task of this application concern Exam page, because, through this application, a student may select his exam, then submit his answers to be automatically signed, graded, and reported.

Class OdbcToSQL: A Java class including the connection to database through Jdbc/Odbc Drivers.

Class AcYear: Class contains some utile functions concerning Academic Year.

Class Semester: Class contains some utile functions concerning Current Semester.

Class ExamUtils: This class contains some utile functions for the Web Site in general.

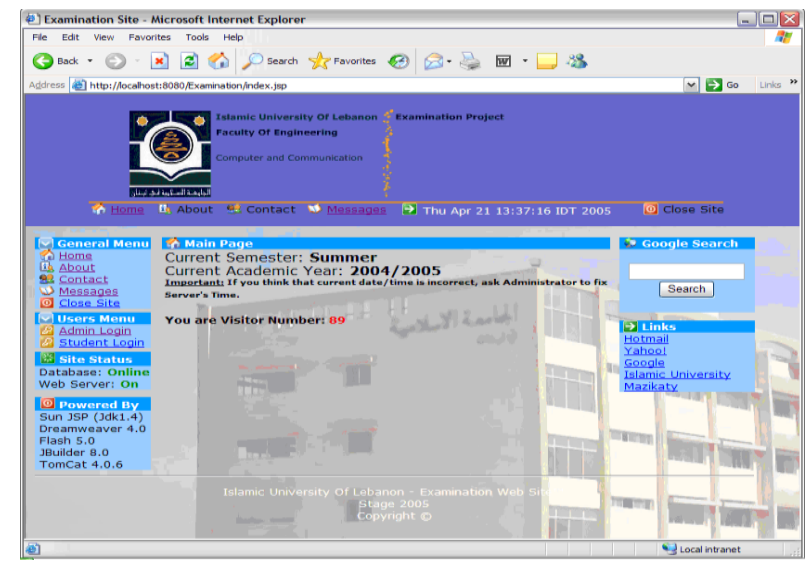

Figure 7. The main form for the Examination system where students can log into the system.

The students can access the exam form by loging into the server using their own user names and passwords previously created by the administrator. When signing in, the student may specify certain information before starting the exam. He must specify the symester, the course name and other information.

When connecting to the exam, all the information about the exam will be displayed for the student students [Figure 7].

The exam is loaded to a certain form by selecting all the questions related to the exam.

The questions will be selected and arranged randomly so that two students making the same exam will have the same questions but with different order to prevent cheating.

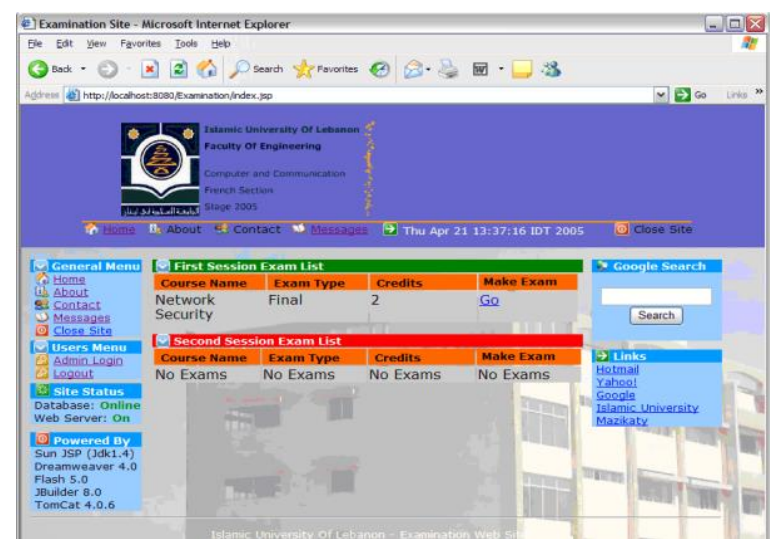

Figure 8. The information form for the requested exam. 
When starting the exam, the counter start counting down, indicating the remaining time for a student to finish his exam.

The exam may contain as much as the teacher needs of questions, the questions are divided into three types. True and false, multiple choice and fill in the blanks.

The questions are loaded randomly as mentioned previously. a student starts answering his questions while the counter is counting down, when reaching to a critical time, the counter starts blinking on and off indicating the end of the exam. When the time is over, all the answer's places will be disabled except the submit button enforcing the student to submit his answers to the server [Figure 9].

When submitting the exam, the answers will be saved in a special temporary table, the answers will be corrected by matching them with the answers provided by the teacher. Then the mark is calculated and displayed on the screen and saved down in the database. The mark is generated with the grade [Figure 10].

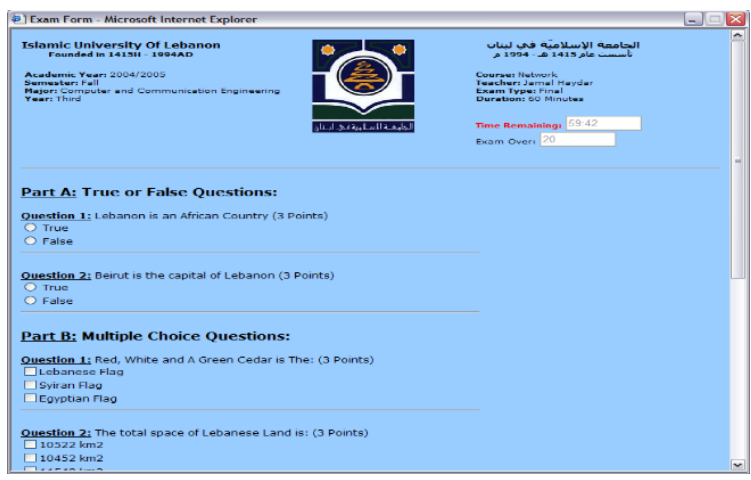

Figure 9. The Exam sheet form where exam process take place, the page contains information about the exam.

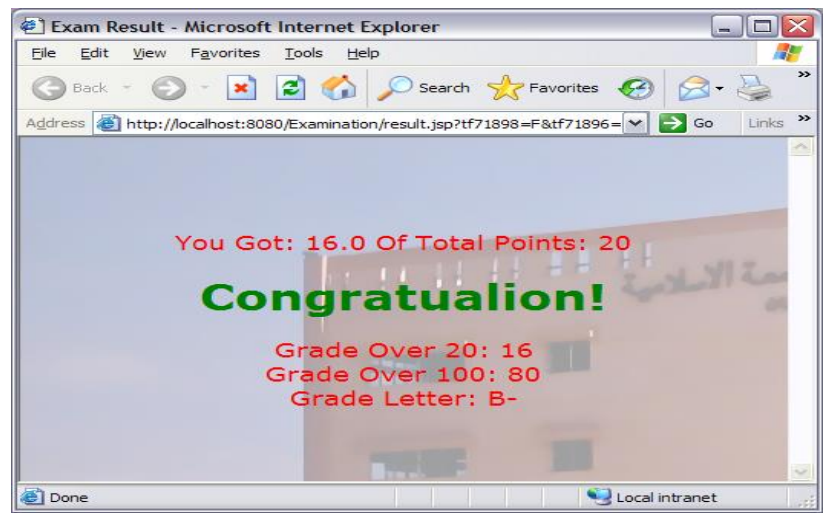

Figure 10. the result form after finishing the exam and submitting all the information to be corrected and obtain the result.

\section{Additional features.}

The system includes the following additional features.

1- A GUI Interface in the Admin Application allowing teachers to load their exams dynamically using buttons and text fields...

2- A Reporting System for the Exam Interpreter, so Administrator can know if the exam loading process succeeded.

3- An Admin Auto-Define System, where first person loads Admin Application will be considered directly "System Admin".

4- A Reporting System allows the student to compare between his answers and the correct ones

5- Adding a picture for every student. 
6- Ability to create questions with pictures or sound clips.

\section{Conclusion.}

The Online Electronic Examination System (OEES) depends on a deep research and good analysis for the need to digitize the exams in order to achieve the maximum benefits of the machine, minimize time and cost and establish accuracy and security and to prevent exam cheating.

The system is implemented using advanced technologies and it achieved it goals.

The system is based on a strong database controlled by a well secured distributed applications.

The system randomizes the creation of exam sheets, so same exam may have many forms for different students.

The system is a real system and was tested successfully. Errors are controlled using error detection and control modules imbedded in the system.

In short, the OEES is an introduction to enormous fields of the E-Learning domains. The E-Learning techniques will support teaching methods and secure exams over the next generations.

\section{References}

[1] Software engineering, a practitioner's approach , 2005.

[2] Database system concepts , Abraham Silberschatz, 2001.

[3] Java : the first semester, Quentin Charatan and Software system development : a gentle, 2001.

[4] Data structures and the Java collections framework, 2005.

[5 Demystifying E-Learning Standards, Harvi Singh.

[6]www.java.sun.com.

[7] www.elearningguild.com.

[8] www.e-learningcentre.co.uk.

[9] www.adobe.com/go/gntray_sol_elearning.

[10] www.elearningmag.com. 